\title{
Magaly GHESQUIÈRE et Laurence MEURANT, École et surdité. Une expérience d'enseignement bilingue et inclusif
}

Presses universitaires de Namur, 2018.

\section{Jean Giot}

\section{OpenEdition \\ Journals}

Édition électronique

URL : http://journals.openedition.org/esp/4199

DOI : 10.4000/esp.4199

ISSN : 2532-0319

Éditeur

Centre d'Information sur l'Éducation Bilingue et Plurilingue

Édition imprimée

Date de publication : 1 juin 2019

Pagination : $99-105$

ISSN : 1127-266X

Référence électronique

Jean Giot, « Magaly GHESQUIĖRE et Laurence MEURANT, École et surdité. Une expérience

d'enseignement bilingue et inclusif », Éducation et sociétés plurilingues [En ligne], 46 | 2019, mis en ligne le 01 février 2021, consulté le 01 mars 2021. URL : http://journals.openedition.org/esp/4199 ; DOI : https://doi.org/10.4000/esp.4199

Ce document a été généré automatiquement le 1 mars 2021.

(C) CIEBP 


\section{Magaly GHESQUIÈRE et Laurence MEURANT, École et surdité. Une expérience d'enseignement bilingue et inclusif}

Presses universitaires de Namur, 2018.

\section{Jean Giot}

\section{RÉFÉRENCE}

École et surdité. Une expérience d'enseignement bilingue et inclusif. Presses universitaires de Namur, 2018. ISBN: 978-23-902-9005-6

Ce livre «vise à transmettre ce que l'expérience de presque vingt ans a permis à l'équipe enseignante d'apprendre sur la pédagogie bilingue à destination d'EMS [élèves malentendants et/ou sourds]: ses enjeux, ses contraintes, les questions qu'elle pose, les aménagements qu'elle implique et les compétences qu'elle requiert »(p. 11), des classes maternelles aux classes terminales du secondaire, en Belgique francophone. Cela, sur une trame instruite, et qui se lit tel un récit où rigueur et invention ouvrent à ce qui est en cours, à l'hétérogénéité des acteurs, à la singularité des histoires.

Quel lectorat? Enseignants bilingues ou non, personnes s'adressant à des EMS, interprètes, et tout public qu'intéressent des sujets de pédagogie, de linguistique, de traduction, voire d'anthropologie. Le lecteur gardera en mémoire ce que lui indique l'introduction: il existe plusieurs langues des signes [LS] de par le monde, et celles d'usage parmi les Sourds vivant dans des communautés de langue vocale française diffèrent entre elles (p.ex., la LSF - langue des signes française - n'est pas la LSFB langue des signes de Belgique francophone - sans que cela gêne l'intercompréhension; de même qu'en toute communauté linguistique, il y existe des variantes, p.ex. 
géographiques ou de générations, sans que cela empêche l'existence d'une koinè ou l'intercompréhension).

3 « Inclusif »? L'inclusion est distincte de l'intégration, qui laisse à l'élève le souci de s'intégrer à l'environnement proposé et par là, souvent, écarte de fait la LS. Dans le dispositif présenté, des groupes d'enfants sourds sont inclus dans des classes d'enfants entendants. Cette groupalité permet que la LS et la langue vocale circulent entre pairs, entre élèves et adultes, et entre adultes, dans des circonstances naturelles. L'inclusion mise en œuvre se rapproche du dispositif dit de "co-enrollment». Un chapitre introductif décrit ce qui rapproche et ce qui distingue l'expérience que le livre relate, de pratiques et de dispositifs bilingues autres - il se complète d'une riche bibliographie. Parmi ses spécificités: français et LSFB traitées avec équité et enseignées avec la même richesse aux EMS; les classes dirigées par deux co-titulaires, ou binôme, dont l'un est bilingue français-LSFB et spécialiste en surdité - il a la responsabilité des EMS, et l'autre enseignant celle des $\mathrm{EE}$ [élèves entendants]; disponibilité d'interprètes; la formation des enseignants assurée par transmission entre eux et en lien avec une association et avec l'université; les élèves sourds minoritaires dans une proportion représentative de la réalité sociolinguistique ambiante - ce qui contribue à les familiariser avec elle et à faciliter leur autonomie, tout en évitant une ghettoïsation.

4 «Bilingue »? D'un bilinguisme bimodal (avec les nuances fonction des profils d'élèves) puisqu'entre une LS non écrite et une LV [langue vocale] écrite. Au terme du secondaire, il est attendu que l'EMS puisse «écouter un message en LSFB et être capable d'en transmettre une version totale ou partielle en français écrit (correspondant le plus possible au niveau attendu d'un EE); lire un texte en français pour en donner une version en LSFB; produire un texte en français ou en LS pour créer ensuite son équivalent dans l'autre langue » (p. 259). Dès lors, l'élève apprend qu'en passant d'une langue à l'autre il est nécessaire d'adapter le message au système de l'autre langue: prise de conscience métalinguistique, renforçant les acquis par leur transfert d'une langue à l'autre, simultanément à une perception de son propre profil linguistique.

5 Ce bilinguisme est un objectif (LSFB et français sont langues enseignées) et une modalité pédagogique (ce sont langues d'enseignement), sans mélange, mais dans une alternance explicite et réfléchie, ouvrant non sur une juxtaposition de monolinguismes mais sur une hétérogénéité évolutive (selon les individus) des compétences en langues. Ainsi cultivée, la LV, qui s'impose en maintes situations, ne compromet pas pour l'EMS une " réassurance narcissique par affiliation » (p. 33) puisque, d'une part, elle n'exclut pas la modalité visuo-gestuelle entre pairs (voir p.ex. préface du Dr. B. Drion à P. Bélissen et al., Paroles de Sourds, P., La découverte, 2018) et, d'autre part, elle favorise une intelligence de différences culturelles et l'étude d'une langue supplémentaire (ici l'anglais).

6 Aussi l'enseignant bilingue doit-il « être capable de distinguer les apprentissages qui sont spécifiques à une langue et à une culture, et ceux qui ne le sont pas; [et...] d'amener les élèves à s'approprier de nouveaux savoirs par le biais d'une langue ou d'une autre, même lorsqu'il s'agit de savoirs propres à une seule des deux cultures » (p. 84). Cette translation toujours réinventée entre langues s'illustre notamment dans un principe central de la pédagogie, appelé triple relation.

7 «Cette appellation souligne que l'alternance linguistique se construit dans une triangulation avec les concepts enseignés. Il est attendu des EMS, en effet, qu'ils 
acquièrent chaque concept dans les deux langues, qu'ils soient capables de le réinvestir dans les deux langues, et même qu'ils puissent explicitement comparer les deux façons d'exprimer les mêmes concepts [...] L'enseignant construit la triple relation par le recours successif à la LSFB et au français, chacune dans sa structure propre, en les nommant et en les distinguant explicitement. Chaque cours est conçu comme un tissage serré entre les deux langues, les deux cultures, les savoirs enseignés et les compétences sollicitées » (p. 37-38). Exemple: les concepts communs à des situations ou objets différents tels que plus, moins, égal, autant varient peu en français selon que l'on parle p.ex. de quantité de pommes ou de hauteur de tours, alors que ces deux cas se signent différemment en LS. Le concept sera le même, mais le processus pour l'acquérir diffèrera selon les langues. Enseignants et élèves sont sans cesse amenés à construire des liens entre concepts abordés (et notation mathématique, le cas échéant), formulations signées et français écrit. Au vrai, ce mouvement reproduit à son niveau celui qui permet aux langues dites naturelles de s'organiser: les langues «s'écartent de l'expérience immédiate, pour pouvoir, en retour, mieux la nommer» (P. Judet de la Combe et H. Wismann, L'avenir des langues, P. Cerf, 2008, p. 82). Ainsi ce travail à l'Ecole est-il profond, infiniment.

Du coup, il est apparu que l'élève tire bénéfice d'un cours qui le soutienne dans l'exercice du passage d'une langue à l'autre, lequel constitue aussi un exercice de la vie quotidienne. Un cours de bilinguisme a été créé, qui renforce les acquis par transferts entre langues, et qui aide à utiliser fonctionnellement la triple relation. Exemples sur les mots très fréquents ou sur les modalités sémantiques (pouvoir, devoir), p. 181-182. L'enseignant signant devra pouvoir établir un diagnostic des difficultés en fonction de chacun des axes de la triple relation, et donc savoir introduire un concept, au besoin, par une contextualisation différente de celle utilisée par l'enseignant francophone. Exemple: l'hyperonyme instrument de musique, p. 88, ou le couple pair-impair p. 242. Ce qui implique chez l'enseignant bilingue, et pour partie chez le binôme d'enseignants, un recul analytique sur chaque langue et sur leur comparaison. Ce regard distanciant fait peu à peu percevoir la puissance expressive et inventive de ces langues, devenant, en ce contexte d'abord, langues « patrimoniales » (P. Frath, communication au colloque de l'OEP [Observatoire européen du plurilinguisme], Paris, novembre 2018). «Ce mouvement est dialectique au sens où il pose d'abord l'écart, comme nécessaire, comme condition initiale d'un rapprochement [...] Le 'propre' dans 'réappropriation' n'est pas donné, il est à découvrir »(P. Judet de la Combe et H. Wismann, op.cit., p. 138).

Il se conçoit que l'ouvrage consacre un chapitre à réfléchir à des problématiques liées au langage (ouie ou cognition p.ex.) - ainsi aux conséquences du fait que les champs perceptifs de l'enfant sourd et de l'enfant entendant se multiplient ou se réduisent différemment. Mais surtout: «En se focalisant sur la prétendue opposition entre le développement $d u$ langage oral et l'acquisition d'une langue signée, le discours ambiant détourne l'attention des parents de l'urgence du développement linguistique de l'enfant, à un stade pourtant crucial de son développement. [...] Installer chez l'enfant une première langue, quelle qu'elle soit, est capital pour son avenir» (p. 104). Un déficit expérientiel lié à une carence linguistique a des effets sur le développement cognitif, sur la capacité à s'adapter à des situations nouvelles, ou à imputer à des tiers des représentations par définition inobservables. A la lumière de telles aptitudes, on voit qu'il serait inadéquat de réduire les enjeux de l'accès au langage à une capacité à entrer dans l'écrit (comme l'imagine souvent une conception naïve de l'entrée en scolarité). L'immersion dans un bain linguistique riche, cohérent et redondant, par 
quelque langue que ce soit, façonne les compétences, variablement selon que l'entourage sait s'adapter à la surdité. Quatre profils d'EMS sont présentés, et suivis dans leurs différences au fil des exemples et des niveaux d'études: français langue première, LS langue première, bilinguisme à l'entrée à l'école, déprivation de langue avant l'âge de trois ans, où l'effort premier est de (re)conduire l'enfant au réflexe et au désir de chercher à comprendre. Exemples: stades d'apprentissage de la lecture p. 158 ou lecture d'un tableau à double entrée p. 79. Autant de petits romans de formation.

En conséquence, a) il est illusoire de trouver une méthode adaptée à l'ensemble de ces divers profils d'EMS: les chapitres consacrés à la lecture et à l'écriture suggèrent des pistes d'intervention par profil (exemple: comment transférer sur la langue seconde de l'élève le fait qu'il aura reconnu dans sa langue première signée l'existence d'unités indécomposables). Le détail porte tant sur l'accès à une conscience phonologique du français que sur le vocabulaire, la morphosyntaxe, les constructions discursives ou le travail métacognitif de comparaison des langues; b) l'accès à l'écrit s'étend sur un temps plus long pour les EMS que pour les EE. Dès lors, il est inadéquat d'organiser toutes les activités de français en inclusion avec le groupe des EE: les cours de langue (français, LSFB, anglais) sont organisés majoritairement hors du groupe d'inclusion. Le groupe des EE avance selon les besoins et l'hétérogénéité qui lui sont propres, et pour lui un bilinguisme LV-LS n'est pas au programme - ce qui n'empêche pas un développement de l'empathie chez les EE; c) les outils employés en classe sont conçus en équipe; cahiers, synthèses et vidéos constituant la trace des progressions des élèves se transmettent d'année en année. Qualité du dispositif pédagogique et attention à chaque enfant sont cruciales, a fortiori quand des retards se sont accumulés.

11 Les exemples fournissent en fait de mini-récits mettant en scène obstacles et adjuvants avec finesse dans l'analyse de la complexité et des progressions. Ainsi, pour l'acquisition de l'écrit chez l'EMS, du choix et de l'usage d'outils comme l'alphabet manuel ou une synchronisation avec la labialisation (p. 155). Ou le dialogue avec l'entourage de l'enfant, spécialement familial, capital en classe maternelle, dans la conscience de ce qui distingue l'enfant sourd né de parents signants et celui né de parents entendants, dans l'un et l'autre cas notamment sur l'accessibilité d'un bain linguistique, le désir d'apprendre, le rapport à l'écrit, aux histoires et aux styles. Ou, chez l'enseignant bilingue de maternelle, réaliser des activités fonctionnelles proches du quotidien de l'enfant et sur lesquelles mettre des mots (en français et/ou en LS) et faire interagir les élèves (échanger à propos d'une histoire racontée, nommer objets et gestes tout en construisant, détruisant, etc., parler des sentiments, des ressentis, etc.). "Binôme » d'enseignants (l'un bilingue LS-LV, l'autre francophone) ? L'ensemble de la classe, y compris préparations et évaluations, est cogéré, chaque enseignant gardant une responsabilité particulière envers le groupe pour lequel il a été engagé (EMS-EE). Pour que les retombées positives s'en fassent sentir, il faut un temps de pratique conjointe dans la coordination pédagogique, logistique et relationnelle (p.ex. règles de la classe). Sont décrits l'encadrement institutionnel du binôme et les spécificités attendues dans ce cadre de bilinguisme d'objectif et de travail (triple relation, diagnostic des difficultés différenciées, analyse métalinguistique et contrastive des deux langues).

13 Interprète ? "Le champ d'action de l'interprète est limité en situation pédagogique » (p. 89), et l'adéquation de sa place par rapport à celle des enseignants bilingues doit être identifiée. Contrairement au cas des interprètes scolaires, dont les fonctions - 
spécifiques - sont similaires à celles des médiateurs scolaires en France, «il n'a pas été question de modifier la définition du métier d'interprétation»(p. 90) au sein des classes bilingues ici décrites, métier dont le code déontologique repose sur des principes de fidélité au discours (p.ex.: ne pas quitter le fil de son discours pour y ajouter des corrections linguistiques aux productions d'un élève), de neutralité (p.ex. ne pas s'immiscer dans la vie de la classe) et de secret professionnel (p.ex. ne pas échanger avec les parents sur ce qui se passe en classe pour un élève). Cela est plus difficile à faire accepter en situation scolaire qu'en situation d'interprétation de liaison (p.ex. avec une administration), et pose des problèmes fins exposés dans l'ouvrage sur des exemples suggestifs. L'interprète est un facilitateur, "un outil d'accessibilité et d'équité » (p. 94) pour tous les acteurs, mais c'est l'enseignant seul à qui incombe la responsabilité pédagogique (assimilation des concepts et compétences par les élèves) et disciplinaire de la classe. Enfin, l'interprète a un rôle particulier à jouer en partenariat avec l'enseignant bilingue sourd (p. 97) de sorte que celui-ci puisse assumer sa place d'enseignant à part entière (p.ex. l'accessibilité de supports didactiques). Au total, « les classes bilingues inclusives représentent un microcosme de la société dans laquelle les futurs adultes sourds resteront minoritaires et dans laquelle ils seront amenés à faire appel à des interprètes. Il semble dès lors cohérent que l'école apprenne aux élèves quand et comment utiliser leurs services, et que la position des interprètes de l'école soit la plus représentative des professionnels auxquels ils feront appel à l'extérieur du cadre scolaire » (p. 96).

Si riche, cohérent, créatif et progressif qu'il soit, le dispositif ici évoqué ne résout pas toutes les difficultés posées par la surdité, mais un travail en équipe favorise des choix réfléchis sur les objectifs et leur équilibre sur des périodes variables (une semaine, une année, un cycle). Maturation et adaptabilité facilitées par des espaces d'encadrement et de réflexion: un comité de suivi veillant aux objectifs et à la transmission de l'expérience, un groupe de recherche sur des questions d'ordre linguistique associant à l'université les enseignants (de la classe maternelle à celles du secondaire), les interprètes et des linguistes - sourds et entendants.

Inscrit dans ces durées et ces dynamiques de collaborations (en ce sens les réquisits formulés p.ex. dans J-Cl. Beacco et Chr. Tremblay (coord.), Plurilinguisme et éducation, vol. 1, OEP, 2017), le livre inclut, outre une bibliographie, des tableaux et exposés détaillés, de grande précision pédagogique, p.ex. sur l'impératif présent p. 197, la dictée à l'adulte p. 205, les nombres (distincts des chiffres et des numéros), systèmes numériques, multiples et diviseurs p. 228. Sa facture matérielle lui confère une maniabilité excellemment pédagogique (couleurs par chapitre des pages et intertitres). Des images ponctuent le texte avec une finesse souriante, si bien qu'on apprécie d'autant de suivre les profils et les devenirs des élèves. Aussi n'est-ce pas un "produit achevé » qu'on s'y approprierait, mais le cours, qu'on y adopte, d'une construction vive. Ce n'est pas là, avec les savoirs qu'on y éprouve, le moindre bonheur de lecture. 


\section{RÉSUMÉS}

Questo libro intende illustrare quanto alcuni insegnanti hanno imparato, grazie ad un'esperienza ventennale, da una pedagogia bilingue francese-lingua dei segni destinata a studenti audiolesi (EMS): obiettivi - relativi soprattutto al linguaggio e alla cognizione - ostacoli, interrogativi e adattamenti, competenze richieste. Esperienza originale: la classe, d'età compresa tra i 3 e i 18 anni, è cogestita da due insegnanti, uno bilingue francese-lingua dei segni, l'altro francofono, entrambi con responsabilità relative agli EMS e agli studenti udenti. Le due lingue sono materie insegnate (con la stessa ricchezza) e modi d'insegnamento, poiché i corsi di lingua si tengono fuori inclusione nella stessa classe e in inclusione le altre discipline. L'apprendimento del bilinguismo passa attraverso il confronto delle lingue, che strutturano diversamente la cognizione e la pedagogia, per gli studenti e per gli insegnanti. L'interprete ha un ruolo circoscritto. Numerosi esempi sono forniti per diverse lezioni e differenti profili d'EMS (francese prima lingua, lingua dei segni prima lingua, bilinguismo all'entrata, carenza linguistica precedente l'età di 3 anni). Vi si analizza il fatto che una sola delle due lingue sia scritta, come pure sono analizzate le problematiche di un accesso al linguaggio. Vi si descrive inoltre il contesto istituzionale.

The book's aim is to transmit what teachers have learned from twenty years of bilingual teaching of French and Sign Language (SL) to hard-of-hearing or deaf pupils: what is at stake, on a linguistic and cognitive level, what are the constraints, questions and adjustments, the skills required? The experience was original: the class, with pupils aged 3 to 18 , was managed jointly by two teachers, one bilingual in French and SL, the other a French speaker, each respectively in charge of the deaf and the hearing pupils. Both languages, and English, were taught to the same extent and in the same ways, in the same classroom but separately, the other subjects being taught "inclusively". Learning bilingualism is done by comparing languages, which yields different results as to cognition and pedagogy, for the pupils as well as their teachers. The interpreter's role is closely delineated. Many examples are given of the various classes as well as the portraits of hard-of-hearing or deaf pupils: native French or native SL speakers, bilingual upon entering school, language deficiency before age 3... The fact that only one of the two languages is written is also analyzed, as well as the problems governing access to language; the institutional context is described.

\section{INDEX}

Keywords : French/Sign-language Bilingual teaching, pedagogical experiment, native SL speaker, learning bilingualism

Parole chiave : pedagogia bilingue francese-lingua dei segni, esperienza pedagogica, apprendimento del bilinguismo, accesso al linguaggio

\section{AUTEURS}

\section{JEAN GIOT}

Université de Namur (Belgique) 